\title{
Application of Online and Offline Mixed Teaching Combining Virtuality and Reality in Physiological Experiment Course
}

\author{
Ding Yuemin ${ }^{1,}$, Shen Weida ${ }^{1}$, Li Aiqing ${ }^{2}$, Zhang Xiong ${ }^{3}$ \\ ${ }^{1}$ Department of Clinical Medicine, Zhejiang University City College, Hangzhou, China \\ ${ }^{2}$ Gastroenterology Laboratory, Sir Run Run Shaw Hospital, Zhejiang University School of Medicine, Hangzhou, China \\ ${ }^{3}$ Department of Basic Medical Science, Zhejiang University School of Medicine, Hangzhou, China
}

\section{Email address:}

dingyuemin@zucc.edu.cn (Ding Yuemin)

${ }^{*}$ Corresponding author

\section{To cite this article:}

Ding Yuemin, Shen Weida, Li Aiqing, Zhang Xiong. Application of Online and Offline Mixed Teaching Combining Virtuality and Reality in Physiological Experiment Course. Science Innovation. Vol. 9, No. 5, 2021, pp. 200-203. doi: 10.11648/j.si.20210905.14

Received: September 7, 2021; Accepted: September 26, 2021; Published: September 30, 2021

\begin{abstract}
Experimental teaching is a crutial part in the process of talent cultivation in universities. The main purpose of setting up experimental courses in colleges is to train students' ability to operate and solve problems, and promote students' scientific quality of self-motivation, active exploration and innovation. In the past, there were some technical difficulties in moving experimental courses to the Internet. In recent years, with the development of experimental teaching informatization and the sharing of high-quality teaching resources, the online and offline mixed experimental teaching based on "Internet plus" has already been feasible. Physiological experiment is a professional basic course for undergraduates majoring in pharmacy. During the COVID-19 prevention and control period, the teaching team utilized the online high-quality open teaching resources and the virtual simulation experimental platform, and organically combined it with the physical experimental teaching resources, and launched the online and offline mixed experimental teaching practice combining virtuality and reality. The application of the experimental teaching mode not only effectively breaks through the limitations of time and space, so as to expand the scope of the experiment, but also better exercise students' practical ability, cultivate students' inquiry ability, and effectively improve the teaching effect of the course. Under the background that the online and offline mixed teaching mode of virtuality and reality combination has gradually become the new normal of experimental teaching, the exploration of our teaching team can provide references for the in-depth practice of peers at home and abroad.
\end{abstract}

Keywords: Mixed Teaching, Physiological Experiment, Internet Plus, Virtual Simulation

\section{虚实结合的线上线下混合式教学在生理学实验中的应用}

丁悦敏 ${ }^{*}$, 沈伟达 ${ }^{1}$, 李爱清 $^{2}$, 张雄 $^{3}$

1浙大城市学院医学院临床医学系, 杭州, 中国

2浙江大学医学院附属邵逸夫医院消化内科, 杭州, 中国

3 浙江大学医学院基础医学系, 杭州, 中国

邮箱

dingyuemin@zucc.edu.cn( 丁悦敏)

摘要: 实验教学是高校人才培养过程中的一个重要环节, 开设实验课程的主要目的是锻炼学生操作和解决问题的能力, 提升学生自我激励、主动探究和开拓创新的科学素质。过去, 将实验课程搬到互联网在技术上存在一定的困难。近几 
年随着实验教学信息化建设和优质教学资源共享的推进, 基于“互联网+”的线上线下混合式实验教学已经具备了可行性。 生理学实验是我校面向药学专业本科生开设的一门专业基础课程。在新冠肺炎疫情防控期间, 教学团队利用线上优质 的开放教学资源和虚拟仿真实验平台, 将其与实体实验教学资源进行有机结合, 开展了虚实结合的线上线下混合式实 验教学实践。该实验教学模式的应用不仅有效突破时间和空间的限制从而拓展了实验开设的范围, 同时也更好地锻炼 了学生的动手能力, 培养了学生的探究能力, 有效提升了该课程的教学效果。在虚实结合的线上线下混合式教学模式 逐步成为实验教学新常态的背景下，教学团队的探索可为国内外同行的深入实践提供借鉴和参考。

关键词: 混合式教学, 生理学实验, 互联网+, 虚拟仿真

\section{1. 引言}

随着国家对应用型和创新型人才需求的不断增加, 高 校医学类实验教学面临着新的机遇和挑战, 并进行了持续 的改革和发展 [1]。传统的医学实验教学使用的实验对象往 往是整体动物或动物的组织或器官, 实验操作相对复杂, 而学时却非常有限。教师必须在有限的学时指导学生完成 实验, 这成为教学中的最大难点[2]。2013年教育部启动了 国家级虚拟仿真实验教学中心建设工作[3-4], 极大推动了 实验教学信息化建设, 使得优质实验教学资源通过互联网 得到开放共享。2015年国家层面“互联网+”行动计划的提 出使传统教学模式和教育理念发生深刻变革, 慕课和在线 开放课程建设蓬勃兴起。2020年初新冠肺炎疫情下, 教育 部号召全国高校, 积极开展“互联网+”教学活动[5], 短时 间内大部分的理论课教学实现了实质等效的目的。但对于 医学院校的实验课程来说, 网课的实施存在技术上的困难。 可喜的是, 虚拟仿真实验平台及其项目的建设和应用为医 学院校的实验课搬上互联网提供了基础。疫情防控期间, 利用前期建设好的线上优质的慕课资源和虚拟仿真实验 平台, 各高校的实验教学迎来了新的机遇, 关于高校实验 课教学模式的改革和探索正如火如茶开展 [6]。

生理学实验是基础医学教育的重要环节, 需要通过动 物或人体实验来观察生理情况下的机体功能改变, 对于学 生培养动手操作能力, 深刻理解机体的正常功能过程和机 理有重要作用[7]。在“互联网+”时代背景下, 本教学团队 开展了虚实结合的线上线下混合实验教学的探索, 充分利 用开放的虚拟仿真实验平台, 围绕"以学生为中心"的理念, 创建课前、课中、课后 3 个教学环节层层递进、课外和课 内互动互补的教学新模式, 并将其应用于生理学实验的教 学中, 收到良好效果。本文通过具体介绍本教学团队开展 虚实结合的线上线下混合式实验教学面临的问题和对策、 可行性分析、实际应用、效果评价等, 为国内外同行开展 相关的实验教学改革提供有益参考。

\section{2. 开展虚实结合的线上线下混合式实验教学的 问题和对策}

\section{1. 开展虚实结合的线上线下混合式实验教学存在的问题}

把医学院校实验课的一部分内容搬到线上来开展, 从 传统教学思维出发来看, 的确存在诸多问题, 汇集起来主 要有以下几个:
(1) 实验课的开展一般需要特定的实验室、仪器设 备、实验材料如实验对象、实验试剂、实验模型等, 医学 类实验还需接触实验动物、人体标本等, 在不具备这些物 资保障的条件下无法开展正常的实验课教学。

（2）由于大部分高校尚缺乏自主研发的虚拟仿真实 验平台, 网上资源的利用受到限制, 造成学生的使用体验 比较差。

（3）医学类实验课程都属于综合性实践课程, 以培 养学生的动手能力、分析和解决问题的能力为主要目标, 实验课无法纯粹依靠在线讲解来达到教学目的。

(4) 缺乏适合的“互联网+”教学的配套教材。

(5) 缺乏对线上实验课的教学效果的客观评价方法。

（6）由于师生间的空间阻隔, 针对不同学习类型的 学生无法给予个性化教学资料, 不能很好地实施“因材施 教”方法。

以上是高校实验类课程在开展“互联网+”教学时面临的 共性问题。纵观疫情初期各大高校开展在线教学的实施方案, 不难发现各校对实验课一般都作停课处理, 待学生返校后补 课。这也反过来说明对于实验实践类课程是否适合用“互联 网+”教学模式开展这个问题, 主流观点还是非常谨慎和保守 的。正因为如此，探索基于现代信息技术的“互联网+”教学 新方法在实验课中的应用及其效果, 有助于回答疫情下暴露 的一个共性问题, 即实验课是否可以, 或者部分可以通过在 线教学方式开展, 通过和线下实物实验有机融合, 优势互补, 取得和线下课堂同等甚至更优的教学效果。

\section{2. 开展虚实结合的线上线下混合式实验教学的对策}

得益于国内实验教学信息化建设的快速发展和“互联网 +”行动计划成效的集中体现, 线上优质的开放共享的实验教 学资源和虚拟仿真实验平台给高校教师提供了解决策略。

(1) 针对实验课开设特需的场地、设备和材料等问 题, 可通过借助已建的国内外开放性虚拟仿真实验平台来 解决, 有效突破时间和空间的限制, 弥补实验设备、材料、 人员的局限性[8]。

(2) 对于网上资源的利用受到限制, 体验比较差等 情况, 可以从下几个方面着手解决: 一是积极寻找更多更 优的开放性资源; 二是向某几个教学资源和技术最有保障 的企业申请免费开放的虚拟仿真实验项目; 三是积极筹划 虚拟仿真实验平台的自主研发工作。

（3）针对人才培养目标和教学效果问题, 通过设计 和开发新的综合性探究性实验、调整教学大纲和进度表、 拍摄实验教学视频、优化使用虚拟仿真实验平台与信息化 
教学手段结合的教学方法等一系列举措[9], 来提高教学效 果, 达到人才培养的目标。

（4）针对配套教材的问题, 在前期出版的一整套实 验类教材基础上, 开发更多的优质实验, 拍摄高清的操作 视频, 建设适合线上线下混合式教学的新形态教材。

（5）对于如何评价线上实验课的教学效果这一问题, 做好教学活动设计, 借助新实验项目开发和虚拟平台获取数 据, 网络教学辅导实验报告的撰写, 设计教学效果评价量表, 建成有效的形成性教学评价体系, 确保教学效果的提升。

（6）针对不同学习类型的学生, 通过调查问卷获取 学生基本信息, 为不同类型学生提供符合其学习习惯的各 种形式的教学资料, 如视频资料、音频资料、在线测试、 线上操作考核等, 运用多种方式开展因材施教活动。

通过以上举措, 使学生在完成线上实验课的时候有了 较好的体验, 克服时空限制尽可能获取相关的知识, 并结 合线下实物实验来进一步提升教学效果。

\section{3. 开展虚实结合的线上线下混合式实验教学的 可行性分析}

国内开展线上教学实践的第一波热潮出现在抗击“非 典”时期, 与当年的条件比较, 现在我们身处的时代、技 术、观点等多重因素都发生了深刻的变化。由于近年来虚 拟仿真技术和网络技术的飞速发展, 无论是在线课程平台 的数量和技术水平, 还是师生的硬件设备和网络环境都发 生了翻天覆地的变化, 从而使网上虚拟仿真实验的开展和 线上线下混合式实验教学成为可能。

\section{1. 在线课程平台和技术平台}

实施线上实验教学的两个关键因素是在线课程平台 和技术平台。疫情防控期间，共计超过 4 万门慕课和虚拟 仿真实验平台通过近 40 家在线课程和技术平台向全国高 校免费开放, 此举为全国各高校的在线实验教学提供了强 大的技术支持和可靠的解决方案。

基于教育部《教育信息化十年发展规划（2011-2020 年）》规划，经过近十年的建设，目前我国已经建成以“实 验空间”等为主的一系列国家级、省级、校级等虚拟仿真 实验教学平台, 为实施线上实验教学打下了扎实的基础 [10]。以“实验空间”平台为例, 目前平台共享了超过两千 个实验项目 [11], 覆盖面广、适用性强, 有力保障了在线 实验教学的顺利开展。

\section{2. 硬件设备和网络环境}

目前, 我国已基本实现宽带网络的全面覆盖, 人人享 有优质教育资源的信息化学习环境基本建成。通过分析 980份本科生的问卷, 山东大学杨海军等发现学生家庭的 硬件和网络环境基本可以满足在线教学所需的条件。其中, $91.75 \%$ 的学生有电脑, 所有学生有智能手机, 可以满足“互 联网+”教学对硬件的要求。家中有WiFi和手机有流量包月 的学生占比均超过 $80 \%[10]$ 。本教学团队在开课前也对所 有选课学生做了摸底调查, 结果与上述数据相仿, 所有学 生家庭都具备接受“互联网+”教学的软硬件条件。
在充足的在线资源、合适的硬件设备和满意的网络环 境前提下, 本教学团队在经过缜密筹划和精心准备之后, 启动了虚实结合的线上线下混合式实验教学模式的探索, 将其应用于生理学实验课程的教学实践中。

\section{4. 虚实结合的线上线下混合式实验教学模式在 生理学实验中的应用}

虚拟仿真实验平台及其项目的建设和应用为实验课 搬上互联网提供了基础。虚拟仿真实验能够让学生身临其 境般进行实验操作, 有助于加深知识理解、提高学习兴趣、 培养创新精神[12]。但由于这种模拟实验操作还不能完全 取代实际动手操作, 因此, 本教学团队在实验教学模式改 革过程中, 把虚拟仿真实验作为一种很好的辅助手段, 当 其与传统实验教学相结合, 才能为广大师生认可, 并发挥 更好的作用。下面以我院生理学实验这一门实验课程的教 学为例, 详细介绍虚实结合的线上线下混合式实验教学模 式在课程教学过程中的实际应用。

虚实结合的线上线下混合式实验教学模式的设置是 将学习分成课前、课中、课后三个阶段（图1）。（1）课 前阶段: 教学团队前期已经制作完成实验教学视频 28 个, 发布在SPOC在线平台, 让学生通过反复观看视频了解实 验原理、操作过程等基本内容, 并在生-师、生-生之间进 行实验前讨论, 为实验做好准备。(2) 课堂教学阶段: 根据情况可分成“互联网+”线上课堂和线下课堂两种, 线 上课堂可采用直播模式开展线上翻转课堂教学, 结合使用 虚拟仿真实验平台, 让学生进行虚拟操作, 完成结果记录; 线下课堂则通过面授、小组讨论、测试、小组比赛等更灵 活多样的方式进行现场教学, 着重协助学生在实验室完成 相应的操作, 观察实验现象, 探索背后机理, 并答疑解惑。

(3) 课后阶段: 可分为线下和线上两块内容。学生完成 实验报告的书写和提交, 该部分为线下内容。教师通过在 线上平台布置相应的作业, 组织更深层次的讨论, 促进学 生自主探索、创新能力的提高, 这些又是线上的内容。
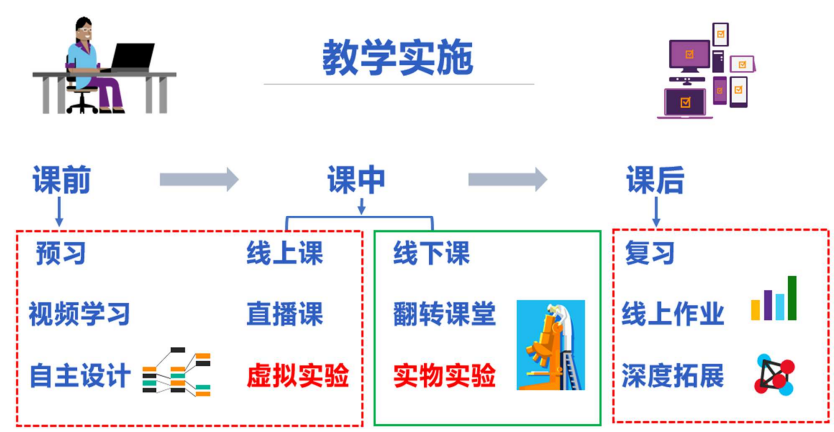

图1 虚实结合的线上线下混合式实验教学模式图。

可以看到, 以上实验教学模式的应用彻底改变了原来 实验教学较为单一的方式, 将原来课堂教学中的理论讲解、 操作演示、小组讨论模块搬到线上提前进行; 而课堂上则 充分发挥翻转课堂的作用, 激发学生积极性和主动性; 配 合实物实验（约占 $60 \%-80 \%$ ）和虚拟仿真实验（约占 $20 \%-40 \%$ ) 操作, 锻炼学生的动手能力, 并培养他们自主、 
探究、创新的素质; 借助虚拟仿真实验不受时空限制、线 上互动和讨论方便、可实现碎片化教学和学习等的特点, 结合课后的拓展性讨论和作业, 达到知识外延的目的。如 此形成的 “以学生为中心”的线上线下混合式实验教学模 式, 有助于激发学生的学习兴趣、培养学生探索知识、解 决问题的能力 [13]。

\section{5. 虚实结合的线上线下混合式实验教学模式的 效果评价}

\section{1. 锻炼动手能力}

虚实结合的线上线下混合式实验教学模式能更好地 锻炼学生的动手能力, 增加学生的学习兴趣和自主性, 帮 助巩固理论知识, 提高分析和解决实际问题的能力, 提高 了学习效率。

\section{2. 拓展实验范围}

虚实结合的线上线下混合式实验教学模式能更广地拓 展实验开设的范围，既突破实验在时间上和空间上的限制， 也打破了部分苛刻实验条件的制约，使得原先一部分因资源、 条件、安全等问题不能做或不敢做的实验成为可能[14]。

\section{3. 提升探究能力}

虚实结合的线上线下混合式实验教学模式可以更灵活 地培养学生的探究能力, 虚拟实验的设计往往涉及行业的前 沿知识, 比实物实验更具有挑战度, 激发学生对学科问题的 持续探索欲望, 点燃学生创新创业的激情。此外, 实物实验 结束后, 学生仍可借助虚拟仿真实验进行反复强化练习, 加 深对实验原理的理解, 强化学生的自主探究素养[15]。

\section{4. 提高教学效果}

虚实结合的线上线下混合式实验教学模式能更大地 提高实物实验的成功率, 学生通过反复的虚拟实验操作训 练, 可熟悉实验具体细节和步骤, 从而减少实物实验中的 误操作, 保障实物实验的成功。此外, 由于师生能共享国 内外优质教学资源, 借鉴和学习教学和学习的新模式, 从 而显著提高教学效果。

\section{6. 结论}

基于“互联网+”教学和虚拟仿真实验平台的虚实结合 线上线下混合式实验教学模式的应用, 很好地解决了生理 学实验教学中长期存在的一些问题, 提高了课程的挑战度, 锻炼了学生的动手能力, 培养了学生的自主探究能力, 显 著提升了课程的教学效果。我们的实践与探索证明, 虚实 结合的线上线下混合实验教学模式是一条可以实现教学 信息化和现代化的有效途径。在该实验教学模式下, 实践 与知识之间得以形成一个不断循环和促进的闭环, 极大促 进学生的学习主动性和自主性, 并促使学生养成勇于实践、 自主探究和求真创新的科学素质。
在建设一流本科课程的目标指引下, 教学团队需持续 研究和优化混合式教学在实验课程中的应用效果, 不断提 升课程的“两性一度”, 培养高质量的应用型医学人才。

\section{致谢}

本文为 2020 年浙江省一流本科课程《生理学实验》(线 上线下混合式一流课程) 和 2020 年浙大城市学院“金课”培 育项目《人体机能学实验I》的阶段性成果之一。

\section{参考文献}

[1] 孙见飞,钟犧修,江帆,等.基础医学实验教学创新人才培养体 系构建与实践 [J].基础医学教育. 2021,23(08):588-592。

[2] 孟洁,朱秀.融合型开放式医学实验教学平台建设 [J].新乡学 院学报.2021,38(06):73-76。

[3] 国家虚拟仿真实验教学项目共享平台 [EB/OL]. http://www.ilab-x.com/.

[4] 王卫国.虚拟仿真实验教学中心建设思考与建议[J].实验室 研究与探索.2013,2(12):13-16。

[5] 教育部.关于在疫情防控期间做好普通高等学校在线教学 组 织与管 理工作的指 导意见 [EB/OL].http://www.moe.gov.cn/jyb_xwfb/gzdt_gzdt/s5987/2 02002/t20200205 418131.html, 2020-02-05.

[6] 马超,曾红,王宏祥.线上线下混合实验教学模式研究 [J].实验 室研究与探索.2019,38(5):185-189。

[7] 刘健翔,丁悦敏,张薇.生理学与病理生理学实验 $[\mathrm{M}]$. 第1版. 杭州: 浙江大学出版社, 2012:2。

[8] 华康民.高等学校虚拟仿真实验教学项目开放共享模式探 索[J].大学.2021,(27):14-16。

[9] 丁悦敏, 王娜, 刘健翔.社交网络工具结合BlackBoard教学 平台在高校生理学教学中的应用 [J]. 科教导 刊.2017,33:127-128。

[10] 杨海军, 张惠萍，程鹏.新冠肺炎疫情期间高校在线教学探 析[J].中国多媒体与网络教学学报.2020,(4):194-196。

[11] 何珊,方枋,刘慧明,等.虚拟仿真实验教学项目建设措施探索 [J].科教导刊.2021,(3):65-67。

[12] 李平,毛昌杰,徐进.开展国家级虚拟仿真实验教学中心建设 提高高校实验教学信息化水平 [J]. 实验室研究与探 索,2013,31(11):5-8。

[13] 刘健翔, 丁悦敏, 姚雪燕.基于学生自主式选择实验的生理 学实验教学改革[J].科教导刊.2016,255:103-104。

[14] 张敏, 刘俊波.对高校虚拟仿真实验教学项目建设的若干思 考[J].中国现代教育装备.2020,(329):10-13。

[15] 熊宏齐. 国家虚拟仿真实验教学项目的新时代教学特征 [J]. 实验技术与管理.2019,36(9):1-4。 